\title{
Evaluation of real time rain-rate on downlink satellite signal attenuation in Abuja, Nigeria
}

\author{
Alhassan Tijani ${ }^{1 *}$, Samson D. Yusuf ${ }^{2}$, Umar Ibrahim ${ }^{2}$, Abdulmumini Z. Loko ${ }^{2}$, \\ Abdullahi A. Mundi ${ }^{2}$ \\ 1. Nigeria Satellite Communication Limited, Abuja, Nigeria \\ 2. Department of Physics, Nasarawa State University, Keffi, Nigeria \\ *Corresponding author: alhassantijani22@gmail.com
}

DOI: https://doi.org/10.37134/ejsmt.vol7.1.4.2020

Received: 02 January 2020; Accepted: 05 April 2020; Published: 07 April 2020

\begin{abstract}
Natural phenomenon and other human activities in the atmosphere have caused serious variations in rainfall which leads to rain-rates that has resulted in the mitigation of signal transmission in satellite communication. This is due to absorption and scattering of the radio waves, resulting in the reduction of the overall link availability. In this study, the cumulative distribution functions (CDFs) for the predicted rain rate and rain attenuation in Abuja, Nigeria was evaluated using the Moupfouma and Chebil models purposely designed for tropical regions. One year data of signal attenuation and amount of rainfall in the study area for 2018 was collected from Nigerian Communication Satellite limited (NIGECOMSAT) and Nigeria Metrological Center (NiMET). The Ajayi (ITU-R P) models alongside with relevant statistics were employed for evaluation of the rain attenuation in the study area. Results show that the highest rainfall value with mean of $128.07 \mathrm{~mm}$ was recorded in August while the lowest value $01.87 \mathrm{~m}$ was recorded in November. The estimated value of rain-rate in the study area were $1.95 \mathrm{~mm} / \mathrm{hr}$ and $5.57 \mathrm{~mm} / \mathrm{hr}$ in August and September with high attenuation value of $11.01 \mathrm{~dB}-19.67 \mathrm{~dB}$ and $15.84 \mathrm{~dB}-18.64 \mathrm{~dB}$ both on $\mathrm{C}-$ band and $\mathrm{Ku}-$ band. The result Show that, attenuation due to rain in the frequency above $10 \mathrm{GHz}$ is much higher than that for frequency of $4 \mathrm{GHz}$. Therefore, as the size of the rain drop approaches the wavelength of the signal, the more it absorbs its strength and the higher the rain rate attenuation on signal. The result is useful for designing highly reliable microwave links in the study area.
\end{abstract}

Keywords: Attenuation, Rain rate, Downlink, Satellite, Modeling

\section{INTRODUCTION}

Rain drops presence along satellite - earth path can be seenas a major cause of signal attenuation at some frequency level specifically above $10 \mathrm{GHz}$ [1].The absorption and scattering of radio waves by rain drops may results in signal attenuation, which degrades the system performance and limits the usage of high frequencies for terrestrial line of sight communication due to reduction of the overall link availability. The severity of rain impairment increases with frequency, and changes with climatic conditions [2].

Sea, land and atmospheric are the vector for energy exchange, and the critical factor of water cycle which is dynamic in nature is rainfall. In the areas characterized by water scarcity monitoring of rainfall plays an important role for the supply of water; and it is the important tool used in water management, agriculture and other important practical areas of applications that are of great concern to human welfare [3]. 
Heavy rain falls is observed during the beginning of March and the end of October each year [4,5]. At frequencies above $10 \mathrm{GHz}$ (ku-band, ka-band, and L-band) satellite communication major effect along the transmission path cause by rain. Rain drops causes weakening of signal strength which is called rain attenuation. Performance of the communication link depends on the size of rain drop, and it absorbs and scatters energy of the radio wave which degrades the reliability of the propagation. Propagation effects are determined by the type of rain, rain rate, frequency, drop size distribution and drop shapes which depends on Rain effects. The basic input for practically all scientific researches that involves hydrological cycle is associated with precipitation $[6,7,8]$. However, it is extremely difficult to accurately measure precipitation due to its intermittent nature, temporal and spatial variation with sensitivity to the conditions of the environment. The most worldwide used devices for an in-situ measurement of duration and intensity of precipitation is rain gauges, especially with Tipping-Bucket rain gauge that has ability to accurately measures the intensity of rainfall from lower-to-intermediates level and can reliably provide remote recording suitability.

The findings from this study have shown that rain drops causes weakening of signal strength which is often called rain attenuation, at frequencies above $10 \mathrm{GHz}$ (ku-band, ka-band, and L-band) satellite communication major effect along the transmission path cause by rain.

\section{MATERIALS AND METHODS}

\section{Materials}

A $0.2 \mathrm{~mm}$ Tipping Bucket with data logger dosimeters made of funnel, funnel spout, pivot, adjustment screw, leveling screw, working base, which measures and storesthe amount of rainfall, each $0.2,0.5,1 \mathrm{~mm}$ 0r 0.01 inch. Regardless of the rainfall intensity it allows rain to flow at a steady rate to the tipping bucket which has gauge sensorsthat were used for collection of rain data, the lever tips of the rain gauge at a preset precipitation fall amount sends an electrical signal to the data logger. For every periodic event, the data logger sends the log of the received data toNigeria Metrological (NiMET) service center using the satellite network, for each tip, the rain gauge collects an amount of water equivalent to $0.2 \mathrm{~mm}$ rainfall. Tips are generated by a reed switch within the rain gauge, and satellite signal attenuation data during the rainfall were obtained from NIGCOMSAT with the aid of a Spectrum analyzer, Hourly rainfall were taken into consideration to evaluate the attenuation of the signal strength during rainy season, from the monitor control system (MCS).

\section{Methods}

\section{Rain-Rate Evaluation}

Result of four months (4) selected out of twelve months rainfall were evaluated using the rain rate model and statistical analysis from the rain accumulation to obtained rainfall rate.

Therefore; the rainfall rate model is the best for both tropical and temperate climates in this study can be expressed as:

$P(R \geq r)=10^{-4}\left(\frac{K_{0.01}}{r+1}\right) b_{e}\left(u\left(R_{0.01-r}\right)\right)$

where $R_{0.01}(\mathrm{~mm} / \mathrm{h})$ is the rain rate exceeded at 0.01 percent of time in an average year, $r(\mathrm{~mm} / \mathrm{h})$ is the rain rate that is exceeded for a fraction of the time, and $\mathrm{b}$ can be approximated by the expression;

$\mathrm{b}=\left(\frac{r-R_{0.01}}{R_{0.01}}\right) \ln \left(1+\frac{r}{R_{0.01}}\right)$ 
where $\mathrm{u}$ in equation (2.1) is a parameter that governs the cumulative distribution of the rain rate slope, and is a dependence of the geographical features and local climatic conditions.

For tropical and sub-tropical localized conditions, $\mathrm{u}$ is given by equation (2.3):

$\mathrm{u}=\frac{4 \ln 10}{R_{0.01}}\left(e^{-\lambda}\left[\frac{r}{R_{0.01}}\right\rceil\right)$

where $\lambda=1.066$ and $\gamma=0.214$. These account for the fact that, the Moupfouma model requires three important terms; $\lambda, \gamma$ and $\mathrm{R}_{0.01}$. Though, the first two terms $\lambda$ and $\gamma$, were appropriately provided. Chebil's model was used to estimate $\mathrm{R}_{0.01}$ since it allows the usage of long-time accumulation of mean annual, $\mathrm{M}$, at the location of interest as presented in equation 2.4 and is seen by Afahakan et al. [9] as the rain rate equation:

$\mathrm{R}_{0.01}=\alpha \mathrm{M} \beta$

where the constants $\alpha$ and $\beta$ are coefficients of regression defined as $\alpha=12.2903$ and $\beta=0.2973$.

\section{Statistical analysis}

No of Rain $\times 24$ hours

Amounth of rainfall (mm)

\section{Signal Attenuation}

Result of Signal attenuation for four months (4) selected out of twelve months were evaluated using Ajayi model and relevant statistics. Therefore the Ajayi [10] model is the under the influence of this experiment as presented by modifying the ITU-R method such that two reduction factors were introduced; the horizontal and the vertical reduction factor. The Ajayi method is then broken down into simpler steps. This method involves several stages, as follows:

Step 1: The height of the freezing rain level is obtained by;

$\mathrm{H}_{\mathrm{fR}}=5.0$ for $0^{0} \leq \theta<23^{0}$

$\mathrm{H}_{\mathrm{fR}}=5.0-0.075(\theta-23)$ for $\theta \geq 23^{0}$

The reduction factors used were the horizontal reduction factors, $\mathrm{r}_{\mathrm{h}}$ and vertical reduction factor, $\mathrm{r}_{\mathrm{v}}$.

Step 2, 3, 4 and 5 remain unchanged, that is, it still maintains the ITU-R procedure of step 6: The horizontal reduction factor, $\mathrm{r}_{\mathrm{h}}$ was estimated using,

$$
r_{h}=\frac{1}{1+l 0.002_{(R) 0.01}}
$$

Step 7: The vertical reduction factor $r_{\mathrm{v}}$ can be similarly calculated using

$r_{v}=\frac{1}{1+\frac{h f R}{5+0.40^{15}}}$

Step 8: The effective path length, Le through the rain can be evaluated from the equation 2.6 and 2.7 above by applying sine rule,

$$
\begin{aligned}
& L e=\frac{\mathrm{rh} \times \mathrm{Lc}}{\cos E} \\
& \tan ^{-1}=\frac{h f r Y v}{\mathrm{Lcyh}}
\end{aligned}
$$


Step 9: The value of specific attenuation $(\mathrm{dB} / \mathrm{km})$ is then evaluated through;

$$
\gamma=\mathrm{K} R^{A}
$$

Step 10: The value of rain attenuation is obtained, using equation (2.13);

$$
A=\gamma L \mathrm{Le} r
$$

The Ajayi mode that is under the influence of this experiment was employed since the data collected for the experiment served as sources of primary data which enable us to adjust the existing rain attenuation models to suit the present environment, and it is designed in such a way as to measure the average signal strength under normal propagation condition and note the signal strength. Then the signal strength is also measured during rainy conditions while taking note of the rain rate value of that rainfall. Then rain attenuation is computed as,

Rain Attenuation $=\left(\begin{array}{c}\text { signalstrengthunder } \\ \text { normalpropagation } \\ \text { condition }\end{array}\right)-\left(\begin{array}{c}\text { signalstrenght } \\ \text { under } \\ \text { rainycondition }\end{array}\right)$

Since this research will be repeated for different rain events while taking note of their different measured rain rate value. The different rain rate values and their corresponding rain attenuation value were will be tabulated.

\section{Relevant Statistics}

Since rain attenuation is recorded hourly, the measurements of the readings obtained were used to analyze the test criteria. The variation of readings of signal attenuation was evaluated using the range as follows

Range $=$ Highest attenuation value - Lowest attenuation value $(\mathrm{dB})$

\section{RESULTS AND DISCUSSION}

The data generated for this study are presented in figures which are subdivided in two (2) sections which include; Activity of the rainfall in the study area, comparison of attenuation rate during the raining day and normal day both on the Ku-band and C-band.

\section{Activity of the Rainfall in the Study Area}

The activities of the rainfall in the study area was obtained from metrological center, and the data was generated using $0.2 \mathrm{~mm}$ Tipping bucket rain gauge, and hourly rainfall was recorded. The reading obtain from this center was tabulated and analyze using relevant statistics and presented in Table 3.1 and Figure 3.1

Table 3.1: Statistics of rainfall accumulation and signal quality on Ku-band and C-band in Abuja from January to December 2018

\begin{tabular}{ccc}
\hline 2018 & Total Volume of & No of rainy \\
Months & Rainfall Per Month in $(\mathrm{mm})$ & days
\end{tabular}




\begin{tabular}{lll} 
Mar & 018.50 & 4 \\
Apr & 039.46 & 6 \\
May & 148.22 & 9 \\
Jun & 184.60 & 13 \\
Jul & 191.10 & 15 \\
Aug & 200.01 & 18 \\
Sep & 073.60 & 16 \\
Oct & 035.60 & 6 \\
Nov & 000.11 & 2 \\
Dec & 000.00 & 0 \\
\hline al volume of & 866.54 & 92 \\
fall Per year & &
\end{tabular}

Table 3.1 shows that Abuja Nigeria usually experience two major seasons - the dry season between (January and December) with value of $(000.0 \mathrm{~mm}$ and $000.00 \mathrm{~mm})$ and the wet season (between May, to October) with value of $(018 \mathrm{~mm}$ and $035.60 \mathrm{~mm})$. For the purpose of our analysis, the dry season was further subdivided into driest (January, February and December) with total volume of rainfall $(005.30 \mathrm{~mm})$, and early rain (March to May) with total volume of rainfall $(206.18 \mathrm{~mm})$ months. The wet season was subdivided into heavy rainfall (June, August, and September) with value of $(649.31 \mathrm{~mm})$ and moderate rainfall (October to November) with value at about $(35.71 \mathrm{~mm})$ months. Rainfall accumulation, rain rate and rain from the result, as expected, rainfall accumulation was lowest between November and February 2018 and in the months of June to September, 2018 is the highest respectively.

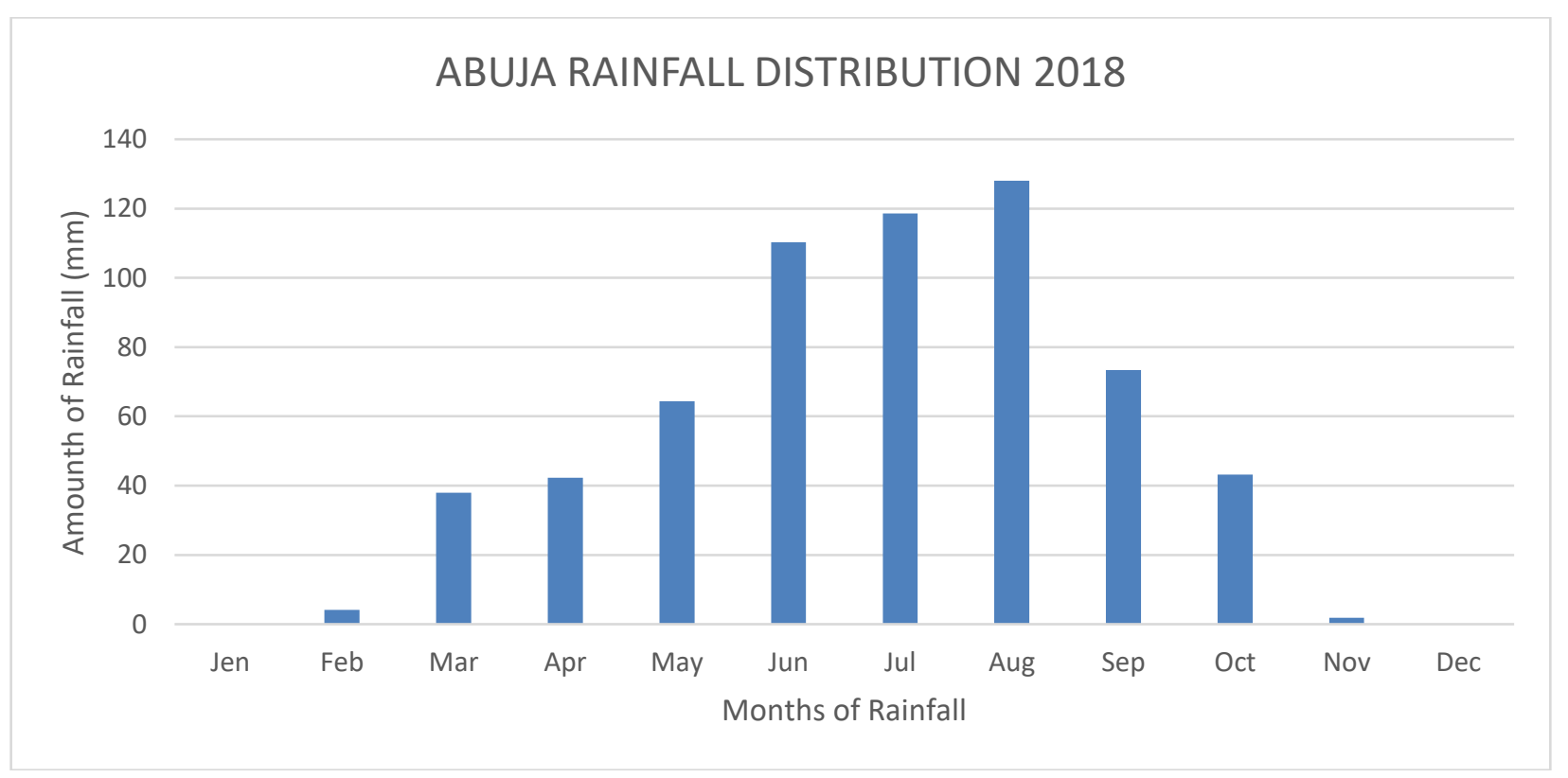

Figure 3.1 Monthly rainfall distributions 
Figure 3.1 shows that Abuja, Nigeria usually experience two major seasons - the dry season between (January and December) and the wet season (between May, to October). For the purpose of our analysis, the dry season was further subdivided into driest (December to February), and early rain (March to May) months. The wet season was subdivided into heavy rainfall (June, August, and September) and moderate rainfall (October to November) months. Rainfall accumulation, rain rate and rain from the result, as expected, rainfall accumulation was lowest between November and February, 2018 respectively.

\section{Comparison of attenuation rate during the raining day and normal day both on the Ku-band and C-band.}

In this; several attenuation rate was recorded, and relevant statistics of range was used to analyze the value with equation (2.1) (Rain attenuation = Signal propagation on rainy day - Signal propagation on normal day) and all the data was run on excel in other to show the correlation and also provide basis for interpolation and proper interpretation of the observation of the signal reception on the raining day and normal day.

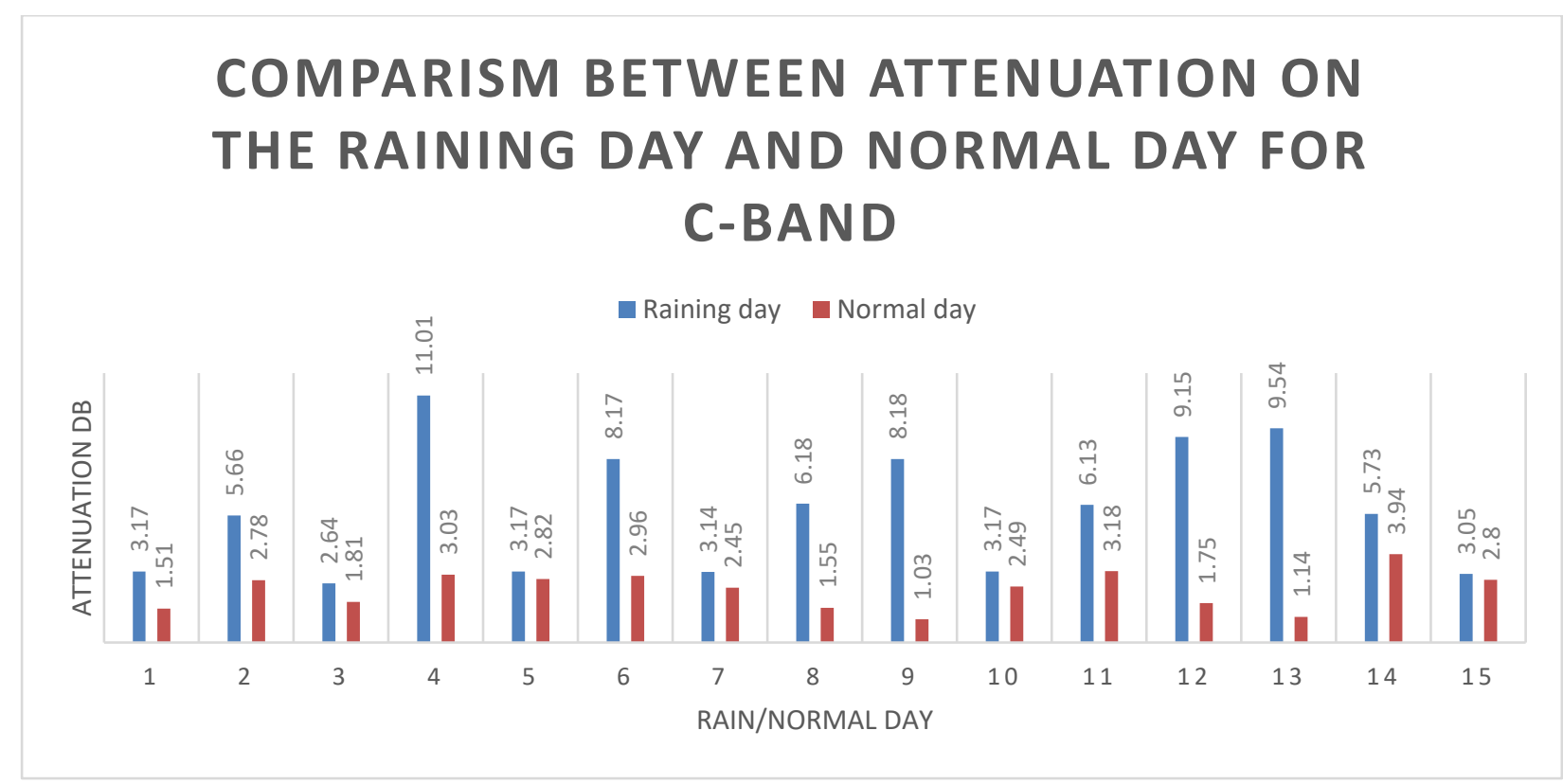

Figure 3.2: showing the reception of signal on the normal day and raining days for C-band, 2018. 


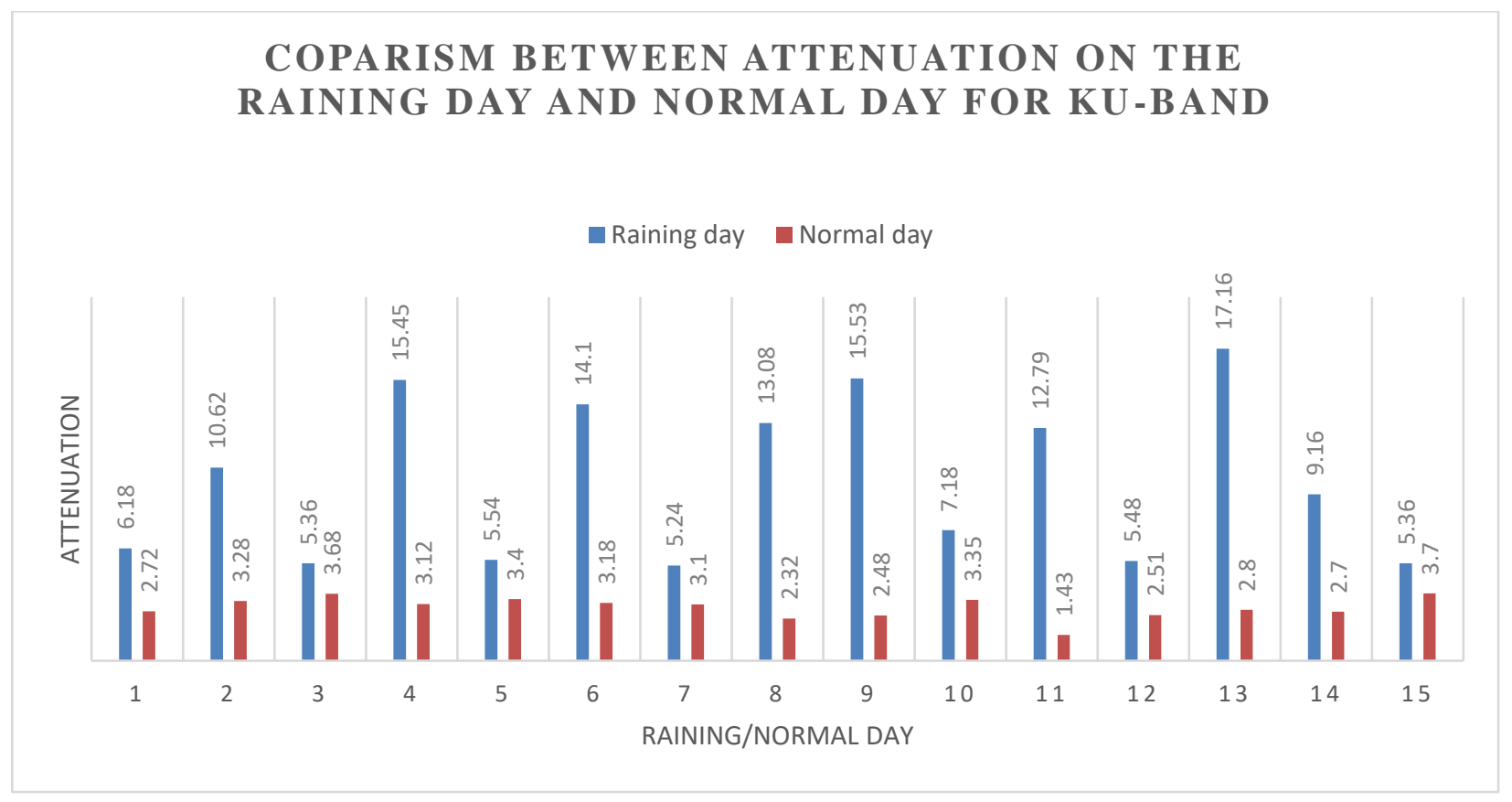

Figure 3.3: Showing the reception of signal on the normal day and raining day for Ku-band, 2018

Figure 3.2 and 3.3 shows the averaged values of signal quality over the locations within the study area during raining days and normal days, the two charts shows that high value of signal quality were recorded during the raining days while low value of attenuation were recorded during the normal day. Figure 3.2 is the attenuation of signal on C-band while Figure 3.3 is the attenuation of signal on Ku-band; the yellow line is for normal day while the blue line is for raining day, on the C-band it shows that the attenuation of signal is high during the rainy day while on the normal day the attenuation is low.

Plate 3.1 and 3.2 shows signal strength on the clear sky and rain-fade effect. Plate 3.1 shows the envelops when both the strength and quality of the signal received falls under clear sky, while plate 3.2 shows the envelop when both the strength and quality of the signal received falls under the effect of rainfade, as a standard. 


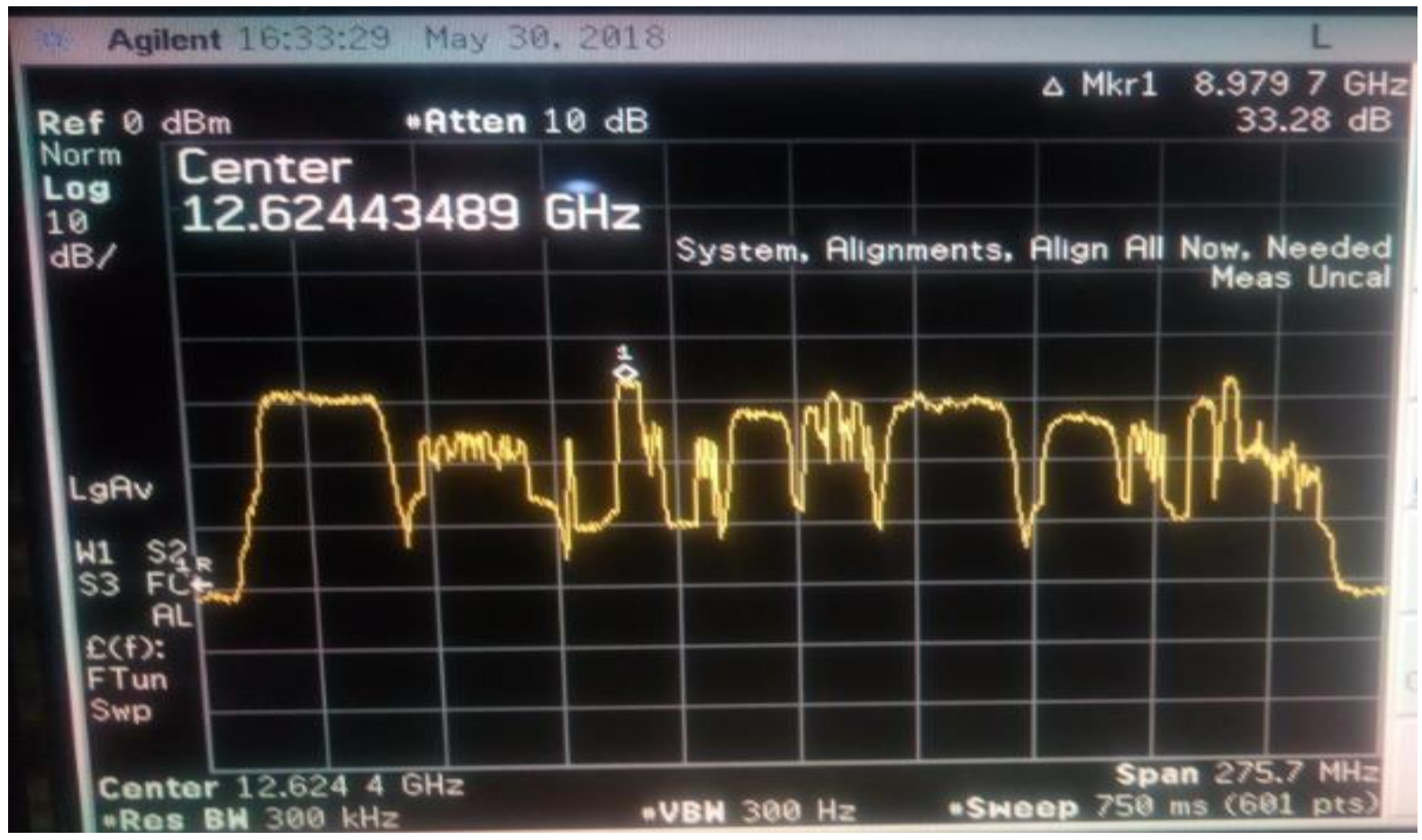

Plate 3.1: Signal from tracking setup showing both Signal strength and Quality on a clear sky

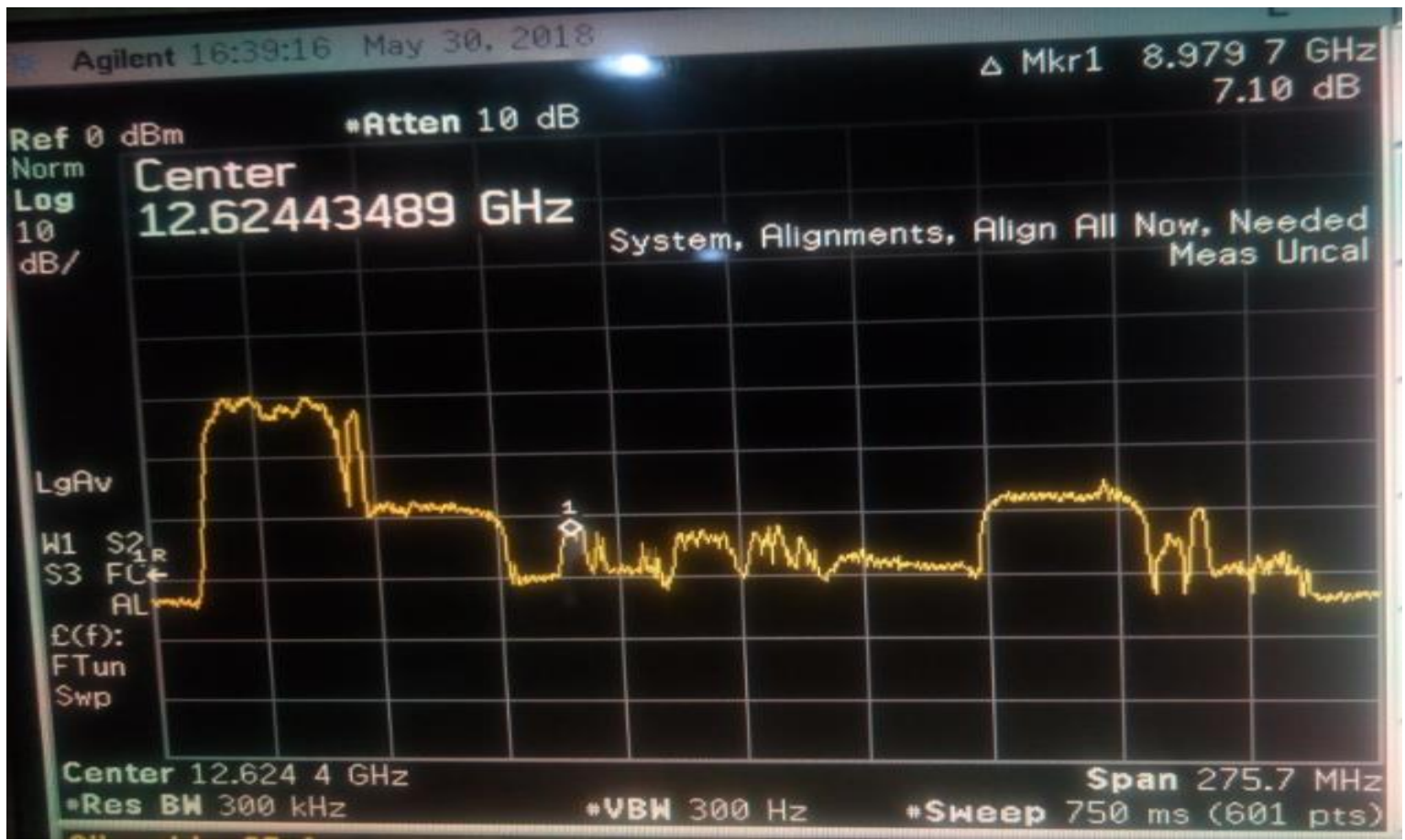

Plate 3.2: Signal from tracking setup showing both Signal strength and Quality on a rain-fade effect

It shows on the view that the tracked signal for C-band and Ku-band has an envelope of analog and discrete spectrum of frequency with signal strength and quality on clear sky and rain-fade effect respectively. It was also observed that in the plate 3.2 that signal react to the intensity of rainfall. The spectrum bands of the tracked received signal under same condition respectfully, it shows that the study area experience high rain fail which lead to more attenuation during the raining days. In plate 3.1 it shows that that the signal quality under normal condition without have a better quality in the study area. 


\section{CONCLUSION}

Based on the results of the analysis activity of the rainfall in the study area, research findings reveal that there is difference between rainfall and rain rate, it also reveals that rainfall accumulation in the year 2018 shows the highest volume of rainfall were recorded in the month of July, August, and September with volume of $796.92 \mathrm{~mm}$ and moderate rainfall is $93.56 \mathrm{~mm}$ and least rainfall is $5.41 \mathrm{~mm}$.

Base on the analysis comparison of attenuation rate during the raining day and normal day both on the Ku-band and C-band. The findings reveal that there is attenuation rate on the raining days and normal days as indicated in analysis; it also shows that attenuation of signal on the rainy days and normal days has effect both on C-band and Ku-band. It was also reveal that mitigation, distortion of signal become high under the rainy condition as shown in the figure 3.2 and 3.3, under this rainy condition poor signal is observed, in direct to home (DTH), or fixed satellite service (FSS), it is reveal that if the attenuate become high as the rain rate increase signal will go off, therefore rain rate noise, distortion which lead to more effect on the signal.it is reveal that if the attenuate become high as the rain rate increase signal will go off, therefore rain rate noise, distortion which lead to more effect on the signal. Under normal condition without rain as indicated in the figure 3.2 and 3.3 attenuation rate have the lowest value. It reveals that as low as the attenuation rate on this normal signal react to it but lesser than the attenuation on the rainy day.

\section{ACKNOWLEDGEMENT}

The authors of this research wish to acknowledge the contributions of the Faculty of Natural and Applied Science, Department of Physics Nassarawa State University, Keffi, Nigeria Satellite Communication Limited, Nigeria Metrological Center, Abuja, Nigeria.

\section{REFERENCES}

[1]. Emiliani, L. D., Agudelo, J., Gutierrez, E., Restrepo, J.\&Fradique-Mendez, C. (2004). Development of rain-attenuation and rain-rate maps for satellite system design in the $\mathrm{Ku}$ and $\mathrm{Ka}$ bands in Colombia. IEEE Antennas and Propagation Magazine, 46(6), 54-68.

[2]. Choi, D. Y., Pyun, J. Y., Noh, S. K.\& Lee, S. W. (2012). Comparison of measured rain attenuation in the $12.25 \mathrm{GHz}$ band with predictions by the ITU-R model. International Journal of Antennas and Propagation, 2012.

[3]. Giannetti, F., Reggiannini, R., Moretti, M., Adirosi, E., Baldini, L., Facheris, L.\& Vaccaro, A. (2017). Real-time rain rate evaluation via satellite downlink signal attenuation measurement. Sensors, 17(8), 1864.

[4]. Bellerby, T.M., Todd, T., Kniveton, D. \& Kidd, C. (2000). Rainfall estimation from a combination of TRMM precipitation radar and GOES multispecral satellite imagery through the use of an artificial neural network. Journal Appl. Meteor., 39(1), 2115-2128.

Ahmed, K., Shahid, S., Ali, R. O., Harun, S. B.\& Wang, X. J. (2017). Evaluation of the performance of gridded precipitation products over Balochistan Province, Pakistan. Desalination, 1(1), 14.

[6]. Bellerby, T., Todd, M., Kniveton, D.\& Kidd, C. (2000). Rainfall estimation from a combination of TRMM precipitation radar and GOES multispectral satellite imagery through the use of an artificial neural network. Journal of Applied Meteorology, 39(12), 2115-2128.

[7]. Cakaj, S. \&Malaric, K. (2008). Downlink performance comparison for low earth orbiting satellite ground station at S-band in Europe, IASTED. In Proceedings of 27th International Conference on Modeling, Identification and Control, Innsbruck, Austria, (1),11- 13. 
[8]. Abdulrahman, A. Y., Rahman, T. A., Rahim, S. K. B. A.\& Rafi Ul Islam, M. (2010). A new rain attenuation conversion technique for tropical regions. Progress in Electromagnetics Research, 26(1), 53-67.

[9]. Afahakan, I. E., Udofia, K. M., \& Umoren, M. A. (2016). Analysis of rain rate and rain attenuation for earth-space communication links over uyo-akwaibom state. Nigerian Journal of Technology, 35(1), 137 143.

[10]. Ajayi, R. A., Soothill, P. W., Campbell, S.\&Nicolaides, K. H. (1993). Prediction of morbidity in small and normally grown fetuses by fetal heart rate variability, biophysical profile score and umbilical artery Doppler studies. BJOG: An International Journal of Obstetrics \& Gynaecology, 100(8), 742-745. 